\title{
SIMULTANEOUS SELF-SUSTAINED ACTUATION AND PARALLEL READOUT WITH MEMS CANTILEVER SENSOR ARRAY

\author{
S.Z. Lulec ${ }^{1}$, C. Sagiroglu ${ }^{1}$, A. Mostafazadeh ${ }^{1}$, E. Ermek $^{1}$, E. Timurdogan ${ }^{1,2}$, Y. Leblebici $^{3}$ \\ and H. Urey ${ }^{l}$ \\ ${ }^{1}$ Koç University, Istanbul, TURKEY \\ ${ }^{2}$ Massachusetts Institute of Technology, USA
} \\ ${ }^{3}$ École Polytechnique Fédérale de Lausanne, SWITZERLAND
}

\begin{abstract}
Parallel readout of a microcantilever array using single magnetic actuator and a single photo detector for concurrent detection is reported. The system includes MEMS cantilever array designed for different resonance frequencies, optical elements for laser beam shaping and focusing, one detector and feedback electronics, and single broadband actuator for parallel excitation. The cantilevers are made using a simple one-mask fabrication process with embedded amplitude gratings at the tips. A line shaped laser beam is used to illuminate the cantilevers. A single readout photodiode is placed at the first order diffraction beam location on the Fourier plane. The amplified photodiode signal is fed back into the magnetic actuation using a preamplifier and a broadband current amplifier. In this paper, we report for the first time parallel monitoring of the thermal resonance peaks of inherently frequency-multiplexed MEMS cantilevers. We demonstrated simultaneous self-sustained oscillations of seven cantilevers by using a single actuator and detector in air environment. The method is suitable for low-cost multiplexed portable biosensors.
\end{abstract}

\section{INTRODUCTION}

Resonant microcantilevers have been widely used for sensing and imaging applications $[1,2]$. A common technique used in dynamic non-contact and tapping mode AFM's and in resonant microcantilever based biological and chemical sensors is to frequency sweep an external AC signal for observing the resonance frequency and/or quality factor change as a result of the detected target [35]. It is also reported that frequency can be monitored real-time using self-sustained actuation (SSA) without requiring signal generators and complicated electronics [1, $6,7]$. SSA can be obtained by amplifying the thermal noise peak and then feeding it back as the actuator signal after applying a certain amount of phase shift. This technique is also used for self-sustained actuation of AFM microcantilevers and known as auto-tapping mode of operation [8]. Previously, we developed SSA method to monitor the changes in resonant frequency of microcantilevers due to accreted biological mass in liquid media and demonstrated sensing with $0.1 \mathrm{ng} / \mathrm{ml}$ sensitivity and high selectivity for Hepatitis applications and reported in this conference last year $[9,10]$.

While SSA and other methods work well for a single cantilever, parallel array operation has not been demonstrated before. This is the first report of a sensor system to simultaneously monitor an array of cantilevers using one actuator and one detector. The method allows readout and analysis of dense array of sensors on a small chip. Therefore, it can open up new horizons for parallel and real time sensing and imaging applications such as multiplexed diagnostics with small sample volumes or parallel AFM.

Microfabrication of the microcantilever array is explained briefly in the next section. Afterwards, operation principle of the closed-loop SSA system is explained in detail including the experimental setup, which contains optical, electrical and mechanical components. The optical interferometric readout and its inherent nonlinearity play a key role in the oscillator operation and explained in the subsequent section. Finally, experimental results are presented that show parallel monitoring of the multi-cantilever array: Brownian noise peaks obtained without any actuation, broadband white noise actuated spectrum, sine wave sweep spectrum and the resonance frequency peaks of simultaneous SSA of a microcantilever array.

\section{MICROFABRICATION}

Cantilevers are fabricated using single mask process, $\sim 1 \mu \mathrm{m}$ thick Nickel was electroplated on a thin Gold layer followed by wet etching for releasing $[7,10]$. Silicon and Nickel surface quality is not very critical as the measurements are made in dynamic mode, which can reject large bias signals. Figure 1 shows different tail length cantilevers with $2-3 \mu \mathrm{m}$ wide grating slits at the tip of the cantilevers to facilitate interferometric readout. The cantilevers are made with different tail lengths to have different stiffness values, leading to different resonant frequencies. Whereas, the cantilever tips are designed to be on a straight line for simultaneous illumination with a $1 \mathrm{D}$ focused laser line.

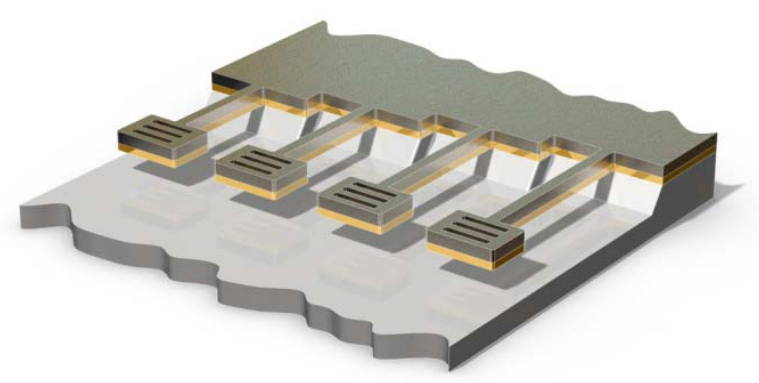

Figure 1: Concept drawing of electroplated Nickel cantilevers with Gold seed layer on Silicon substrate.

Fabricated microcantilevers are shown on a MEMS die in Figure 2. The die $(10 \mathrm{~mm} \times 10 \mathrm{~mm})$ contains thousands of microcantilevers. Adjacent eight cantilevers in the same row are selected and illuminated with a laser line for parallel SSA oscillation. 


\section{OPERATION PRINCIPLE}

The overall system schematically is shown in Figure 3. The system includes a MEMS chip containing the microcantilever array, a coil for actuation, interferometric optical readout setup (detailed in the next section) and closed-loop control electronics. CCD camera and white light source are not essential and for monitoring purposes only. Spectrum analyzer is used to monitor the spectrum.

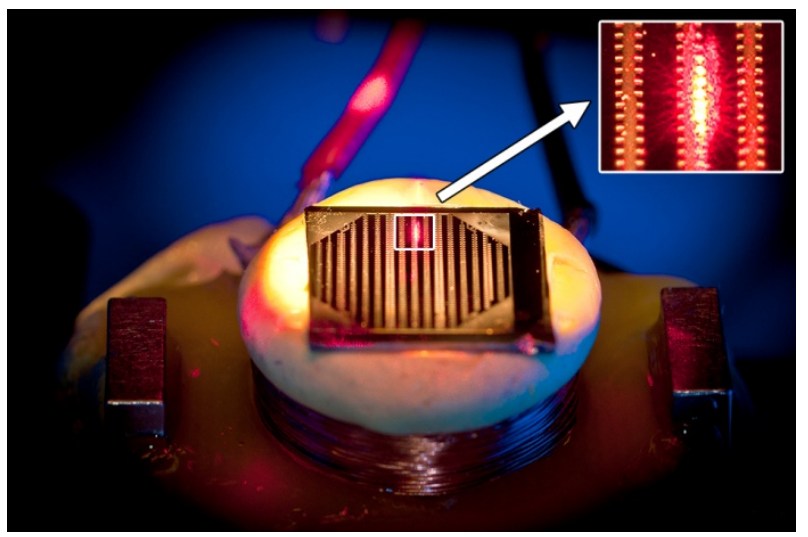

Figure 2: Laser line illuminating the cantilever array on the chip. The magnetic actuation coil underneath and magnets on both sides are visible around the chip.

The microcantilever array contains eight microcantilevers with increasing resonance frequency in the $60-130 \mathrm{kHz}$ frequency range. The microcantilevers are above the coil, which is placed in between two magnets for magnetizing the cantilevers in the direction perpendicular to the anchor, thus leading to an enhanced magnetic force sensed by the cantilevers. The details of the optimized magnetic actuator were reported in [11]. In the feedback loop, the photodetector output is amplified and phase shifted by a variable gain amplifier and a variable phase shifter and fed back to the actuation coil driver. The amplifier and the phase shifter need to be broadband in order to support a range of distinct resonant frequencies within the cantilever array. One advantage of the proposed method is that magnetic actuation and optical readout provide a non-contact/remote actuation and sensing.

Oscillations start with Brownian motion and amplified with a gain of $G$ and $90^{\circ}$ phase shifted by the feedback control system to lock at the resonant frequency of each cantilever, which can be precisely monitored simultaneously using a spectrum analyzer. For a single cantilever case, assuming sinusoidal oscillation characteristics at steady-state, the equation of motion of the system can be written as follows:

$$
m \ddot{x}+b \dot{x}+k x=F_{i n}
$$

where $\mathrm{m}, \mathrm{b}, \mathrm{k}$ are the effective mass, total damping, spring constant and $\mathrm{x}$ is the deflection of the cantilever, which can be expressed as:

$$
x=A e^{j \omega t}
$$

For self-oscillating system the input is given as $j G x$ and Eq. 1 becomes:

$$
-m \omega^{2} x+j b \omega x+k x=j G x
$$

where $\omega$ is the oscillation frequency of the cantilever. At resonance, for zero effective damping, critical gain is calculated as $G_{c}=\omega_{o} b$, with:

$$
\omega_{0}=\sqrt{\frac{k}{m}}
$$

In theory, by making $G=G_{C}$, one can obtain SSA and oscillations grow without bound if there is no nonlinearity that limit the response. In practice, $G$ is taken as a little above $G_{C}$ and oscillation amplitude reaches to a saturation value due to a required nonlinearity in the system and stabilizes. This saturation mechanism forces the SSA oscillation to stabilize at specific amplitude and therefore determines the amplitude of oscillation [12].

SSA is also reported as self-excitation, a method to virtually cancel out the damping in the system and results in sustained oscillation $[13,14]$. For the multi-cantilever case one gain stage is used in the electronic feedback circuit, which can provide necessary gain even for the highest resonance frequency cantilever.

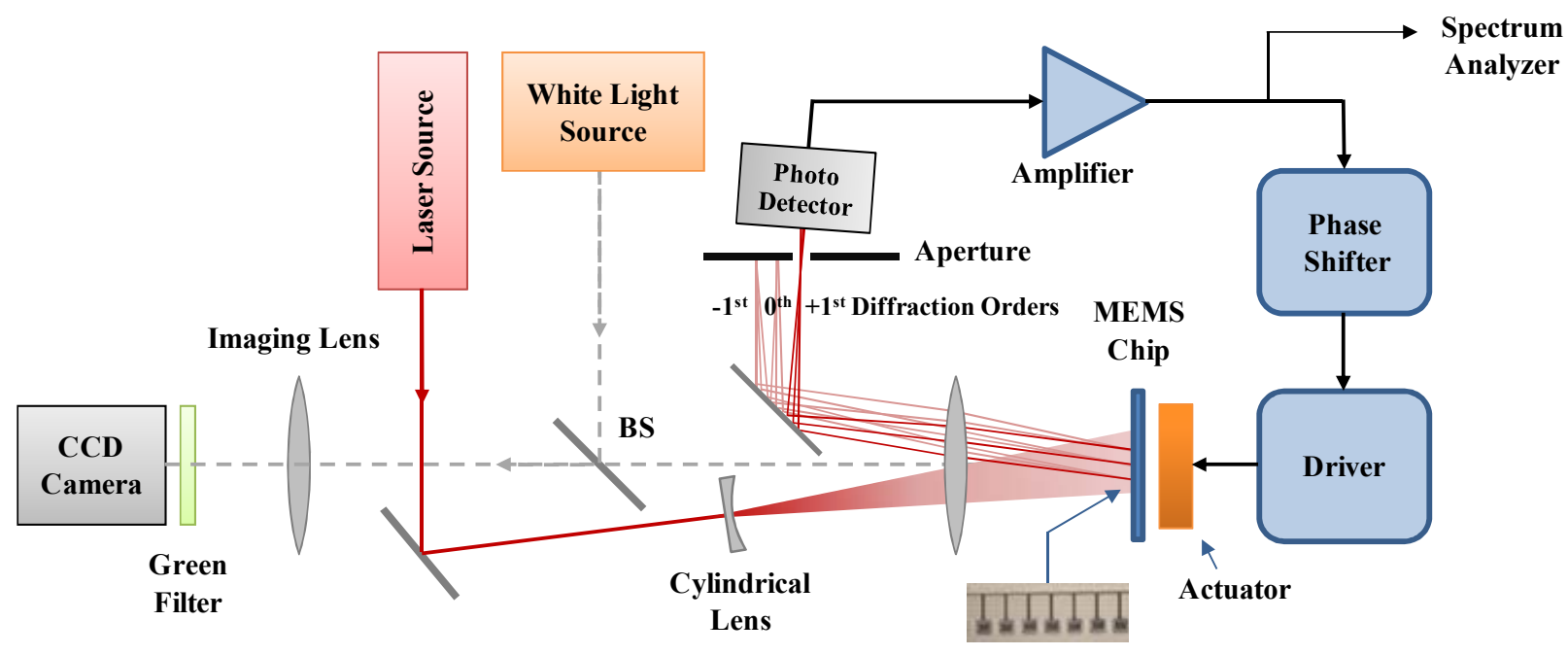

Figure 3: Schematic diagram of the system. 


\section{INTERFEROMETRIC READOUT}

Reflected laser light from the substrate and the sensor surface interfere and create diffraction orders. The photodiode is aligned on the Fourier plane and on the $1^{\text {st }}$ order spot's location. A single photodetector is sufficient to detect the $1^{\text {st }}$ order light from multiple cantilevers. In addition, responsivity of each cantilever depends on the gap underneath each cantilever but the frequency of oscillation is not affected by the gap, variations due to microfabrication, refractive index variations, or environmental vibrations, making the sensor readout robust.

The laser source used for the optical readout is a low power single mode He-Ne Laser. The Gaussian beam shape of the laser is turned into an elongated elliptic shape using a cylindrical lens and then focused on the cantilever tips. The focused laser line is constructed long enough to keep eight cantilevers in the middle of the beam to illuminate the microcantilevers as uniform as possible. Reflected light from the microcantilever surface and substrate interfere and create diffraction orders for each microcantilever. At the Fourier plane, the reflected laser beam separated into its diffraction orders and at this plane it is possible to select the $1^{\text {st }}$ order beams of all the microcantilevers, focused at a point, By aligning the photodetector at this location. $1^{\text {st }}$ diffraction order beam is selected for the readout to avoid large bias in the $0^{\text {th }}$ order direct reflection beam, and also for its higher power compared to the higher diffraction orders.

For alignment and monitoring the microcantilevers a CCD camera, a white light source, a beam splitter and an additional imaging lens is used. The front imaging lens is employed also as the focusing lens for the monitoring optics.

The interferometric readout is based on the Optical Phase Difference in between the reflected light from the microcantilever surface and from the substrate, which can be approximated as twice the gap underneath the microcantilevers. Sinusoidal shaped interference characteristic depends on the wavelength of the readout laser beam and the gap underneath the microcantilever. The normalized intensity at the photodetector can be written as follows:

$$
I_{P D}=\sin ^{2}\left(\pi \frac{2}{\lambda} d\right)=\frac{1}{2}-\frac{1}{2} \cos \left(2 \pi \frac{2}{\lambda} d\right)
$$

where $\lambda$ is the wavelength of the readout laser beam and $d$ is the gap underneath the microcantilever. $d$ can be expressed as:

$$
d=g_{0}+A \sin \left(\omega_{0} t\right)
$$

where $g_{0}$ is the initial gap, $A$ is the deflection amplitude, and $w_{0}$ is the oscillation frequency. Figure 4 illustrates $I_{\mathrm{PD}}$ assuming $\lambda=635 \mathrm{~nm}$ for different $g_{0}$ values, which determine the bias point. In the figure, assuming $20 \mathrm{~nm}$ sinusoidal deflection, two intensity signals are plotted for two different initial gaps at $n \lambda / 2+\lambda / 8$ (marked with red star) and $n \lambda / 2+3 \lambda / 8$ (marked with blue square), where $n$ is a nonnegative integer. Even though interferometric readout does not change the oscillation frequency, optical nonlinearity adds higher harmonics to the system, which will be mechanically filtered by the microcantilevers.

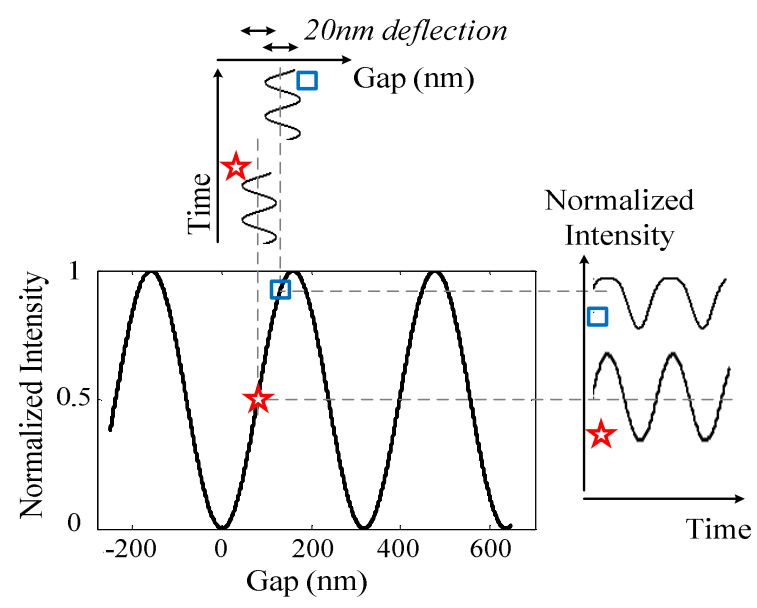

Figure 4: Interferometric readout photodetector normalized intensity characteristic assuming $635 \mathrm{~nm}$ wavelength and $20 \mathrm{~nm}$ deflection for two different nominal gap positions.

\section{EXPERIMENTAL RESULTS}

Multi-cantilever simultaneous SSA and parallel readout concept is experimentally proved by using the system shown in Figure 3. In order to verify the operation of the optical setup and to detect the resonance frequency and quality factors of the microcantilevers, a cantilever array containing eight cantilevers were illuminated via a line shaped laser without any actuation in the open loop mode, i.e. by breaking the loop just before the actuator driver. The photodetector signal was observed via a spectrum analyzer. In Figure 5, the thermal noise peaks of eight different cantilevers are clearly visible. The resonance frequencies and quality factor of the cantilevers are measured with the help of this data. Open loop frequency sweep and driving the system with broadband noise also gave consistent results with the thermal noise response as illustrated in the figure.

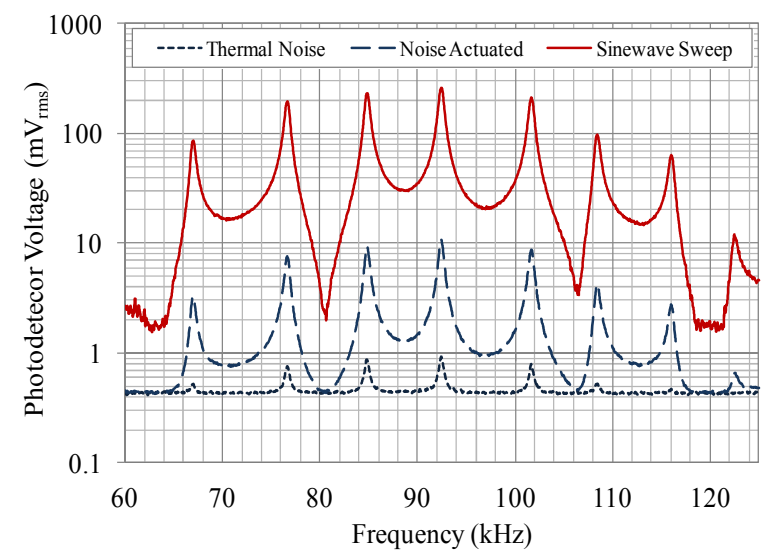

Figure 5: Photodetector signal frequency spectrum that shows thermal noise data (no actuation), white noise driven response and frequency sweep response by coil.

In order to obtain simultaneous SSA, the loop is closed, and in milliseconds the saturation of oscillations were observed and separated SSA oscillations were obtained as very narrow frequency peaks in the frequency spectrum. In Figure 6a, simultaneous SSA oscillations of 
microcantilevers are illustrated. The bandwidth of the oscillation peaks are solely limited by the measurement bandwidth of the spectrum analyzer. Figure $6 \mathrm{~b}$ shows the zoomed-in view of the peak at $92.8 \mathrm{kHz}$ with a measurement bandwidth of around $1 \mathrm{~Hz}$.

a)

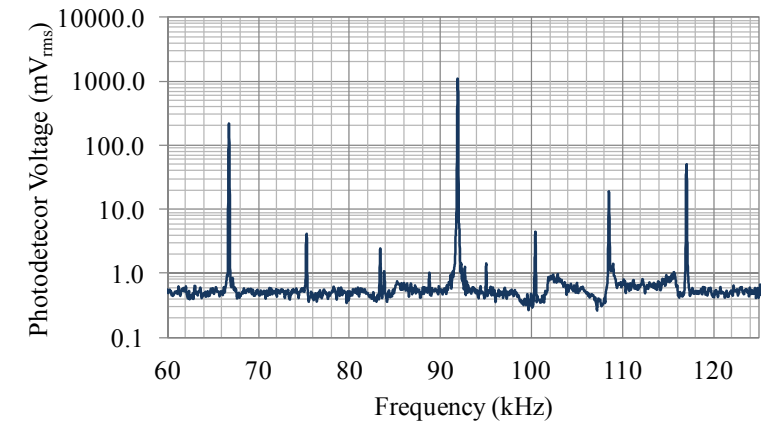

b)

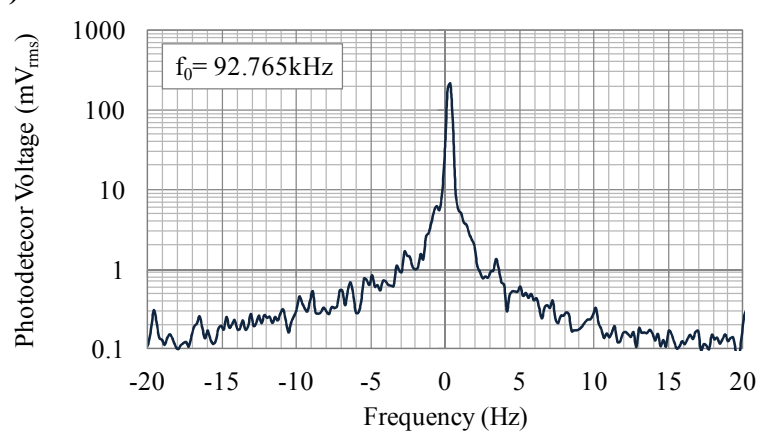

Figure 6: (a) Photodetector signal frequency spectrum that shows simultaneous SSA of MEMS cantilever array. (b) Zoomed spectrum of one of the cantilevers.

\section{CONCLUSION}

A MEMS cantilever array operated with parallel SSA oscillations is reported in this work. A broadband driver circuit and a single driver coil are designed and employed for simultaneous magnetic actuation. A magnet pair is used to enhance the magnetic force by magnetizing the cantilever longitudinally. Microcantilevers are designed with different resonance frequencies and microfabrication was a simple one mask process. In order to perform parallel readout of the simultaneous oscillations, interferometric readout of 8 cantilevers through a single detector and a single electrocoil are used. One set of discrete feedback electronics is utilized to phase shift and amplify the photodetector signal and close the loop.

Without any actuation, Brownian motion of the microcantilever array is observed clearly using a single photodetector and the interferometric setup. SSA oscillations are performed for seven microcantilevers with changing resonance frequencies in the $60-130 \mathrm{kHz}$ range. Measurements are performed in air environment.

Implementation of parallel SSA by using a single actuator and single photodetector opens up new horizons for multiplexed biosensing. The method is suitable for low-cost multiplexed portable biosensor platform with its easy to process sensor array and non-contact actuation and readout mechanism.

\section{ACKNOWLEDGEMENTS}

This work was supported by Tübitak under 109E222 grant. Authors thank to Erdem Alaca and Yasin Kılınç for their invaluable contribution to the project and for microfabrication of the chips.

\section{REFERENCES}

[1] J. L. Arlett, E. B. Myers, and M. L. Roukes, "Comparative advantages of mechanical biosensors," Nature Nanotechnology, Vol. 6, pp. 203-215, 2011.

[2] N. Jalili, and K. Laxminarayana, "A review of atomic force microscopy imaging systems: application to molecular metrology and biological sciences," Mechatronics, Vol. 14, , pp. 907-945, 2004.

[3] P. K. Hansma, J. P. Cleveland, M. Radmacher,D. A. Walters, P. E. Hillner, M. Bezanilla, M. Fritz,D. Vie, H. G. Hansma, C. B.Prater, J.Massie, L.Fukunaga, J. Gurley, and V. Elings, "Tapping mode atomic force microscopy in liquids," Appl. Phys. Letters, Vol. 64, pp. 1738 - 1740, 1994.

[4] S. Ming, L. Shuyou, and P. D. Vinayak, "Microcantilever resonance-based DNA detection with nanoparticle probes," Appl. Phys. Letters, Vol. 82, pp. 3562-3564, 2003.

[5] L. A. Pinnaduwage' A. Wig, D. L. Hedden, A. Gehl, D. Yi, T. Thundat, and R. T. Lareau, "Detection of trinitrotoluene via deflagration on a microcantilever," J. Appl. Phys., Vol. 95, pp. 5871-5875, 2004.

[6] D. Ham, and A. Hajimiri, "Virtual damping and Einstein relation in oscillators," Journal of Solid-State Circuits, Vol. 38, pp.407-418, 2003.

[7] H. Urey, E. Timurdogan, E. Ermek, I. H. Kavakli, and B. E. Alaca, "MEMS biosensor for parallel and highly sensitive and specific detection of hepatitis," MEMS 2011 IEEE Conf., pp. 920-923, 2011.

[8] M. Basso, P. Paoletti, B. Tiribilli, and M. Vassalli, "Modeling and analysis of auto-tapping AFM," IEEE Decision and Control Conference, Vol. 19, pp 5188 - 5193, 2008.

[9] E. Timurdogan, B. E. Alaca, I. H. Kavakli, and H. Urey,

"MEMS biosensor for detection of Hepatitis A and C viruses in serum," Biosensors and Bioelectronics, Vol. 28, pp. 189-194, 2011.

[10] A. Ozturk, H. I. Ocakli, N. Ozber, H. Urey, I. H. Kavakli, and B. E. Alaca, "A magnetically actuated resonant mass sensor with integrated optical readout," IEEE Photonics Technol. Lett., Vol. 20, pp. 1905-1907, 2008.

[11] E. Timurdogan, and H. Urey, "Ferromagnetic thin film cantilevers for sensor arrays," EMSA 2010, Bodrum / Turkey, July 2010.

[12] M. Basso, P. Paoletti, B. Tiribilli and M. Vassalli, "Modelling and analysis of autonomous micro-cantilever oscillations," Nanotechnology, Vol. 19, pp YYY, 2008.

[13] J. Tamayo, M. Calleja, and J. Mertens, "Underlying mechanisms of the self-sustained oscillation of a nanomechanical stochastic resonator in a liquid," Phys. Rev. B, Vol. 76, 180201(R), 2007.

[14] S. Konduri, S. Patra, E. Alaca, and H. Urey, "Micro cantilever sensing in liquid environment with self-oscillation and amplified quality factor," ICMEMS Conference, Paper: OM-O1, Chennai, India, Jan 2009. 